\title{
INVARIANT GRADIENT IN REFINEMENTS OF SCHWARZ AND HARNACK INEQUALITIES
}

\author{
Petar Melentijević \\ University of Belgrade, Faculty of Mathematics \\ Studentski trg 16; 11000 Beograd, Serbia; petarmel@matf.bg.ac.rs
}

\begin{abstract}
In this paper we prove a refinement of Schwarz's lemma for holomorphic mappings from the unit ball $\mathbf{B}^{n} \subset \mathbf{C}^{n}$ to the unit disk $\mathbf{D} \subset \mathbf{C}$ obtained by Kalaj in [3]. We also give some corollaries of this result and a similar result for pluriharmonic functions. In particular, we give an improvement of Schwarz's lemma for non-vanishing holomorphic functions from $\mathbf{B}^{n}$ to $\mathbf{D}$ that was obtained in a recent paper by Dyakonov [2]. Finally, we give a new and short proof of Marković's theorem on contractivity of harmonic mappings from the upper half-plane $\mathbf{H}$ to the positive reals. The same result does not hold for higher dimensions, as is shown by given counterexamples.
\end{abstract}

\section{Introduction and notation}

1.1. Notation. We use terminology and notation from Rudin [7]. Let $\mathbf{B}^{n}$ denote the unit ball in $\mathbf{C}^{n}, \mathbf{B}_{n}$ the unit ball in $\mathbf{R}^{n}$ and let $\mathbf{H}_{n}$ denote the upper half-space in $\mathbf{R}^{n}$. Specially, $\mathbf{H}$ is the upper half-plane in $\mathbf{C}$. By $\mathcal{H}(\Omega)$ we denote the space of holomorphic functions on $\Omega \subset \mathbf{C}^{n}$. The complex scalar product of $z=\left(z_{1}, z_{2}, \ldots, z_{n}\right), w=\left(w_{1}, w_{2}, \ldots, w_{n}\right) \in \mathbf{C}^{n}$ is given by

$$
\langle z, w\rangle=\sum_{j=1}^{n} z_{j} \overline{w_{j}} .
$$

1.2. Bergman and hyperbolic distance. As is well known, the hyperbolic distance on $\mathbf{B}^{n}$ is given by the expression

$$
d(z, w)=\log \frac{1+\left|T_{w}(z)\right|}{1-\left|T_{w}(z)\right|}
$$

where

$$
T_{w}(z)=\frac{\left(1-|w|^{2}\right)(z-w)-|z-w|^{2} w}{1+|z|^{2}|w|^{2}-2 \operatorname{Re}\langle z, w\rangle} .
$$

It is easy to check that

$$
1-\left|T_{w}(z)\right|^{2}=\frac{\left(1-|w|^{2}\right)\left(1-|z|^{2}\right)}{1+|w|^{2}|z|^{2}-2 \operatorname{Re}\langle z, w\rangle} .
$$

For more information on the hyperbolic distance and Möbius transformations, one can consult [1].

https://doi.org/10.5186/aasfm.2018.4324

2010 Mathematics Subject Classification: Primary 30H05; Secondary 31A05.

Key words: $\mathcal{M}$-invariant gradient, Schwarz's lemma, Harnack's inequality, hyperbolic distance, Bergman distance.

The author is supported by MPNTR grant, no. 174017, Serbia. 
We also use hyperbolic distances in some other domains in $\mathbf{C}$ and $\mathbf{R}^{n}$. Let us recall that the hyperbolic distance on $\mathbf{H}$ is given by

$$
d_{\mathbf{H}}(z, w)=2 \tanh ^{-1}\left|\frac{z-w}{\bar{z}-w}\right|
$$

and on $\mathbf{R}^{+}$by

$$
d_{\mathbf{R}^{+}}(x, y)=\log \frac{y}{x}
$$

for $y \geq x>0$. The hyperbolic distances on $\mathbf{B}_{n}$ and $\mathbf{H}_{n}$ are given by

$$
\begin{aligned}
& d_{\mathbf{B}_{n}}(x, y)=\cosh ^{-1}(1+\delta(x, y)), \quad \text { where } \delta(x, y)=\frac{2\|x-y\|^{2}}{\left(1-\|x\|^{2}\right)\left(1-\|y\|^{2}\right)}, \\
& d_{\mathbf{H}_{n}}(x, y)=\cosh ^{-1}\left(1+\frac{\|x-y\|^{2}}{2 x_{n} y_{n}}\right), \quad \text { where } x_{n}, y_{n}>0 .
\end{aligned}
$$

We will use an involutive biholomorphic automorphism $\varphi_{w}: \mathbf{B}^{n} \rightarrow \mathbf{B}^{n}$ given by

$$
\varphi_{w}(z)=\frac{1}{1-\langle z, w\rangle}\left(w-\frac{\langle z, w\rangle}{|w|^{2}} w-\left(1-|w|^{2}\right)^{\frac{1}{2}}\left(z-\frac{\langle z, w\rangle}{|w|^{2}} w\right)\right) .
$$

Note that $\varphi_{w}(0)=w$. An easy verification gives

$$
1-\left|\varphi_{w}(z)\right|^{2}=\frac{\left(1-|w|^{2}\right)\left(1-|z|^{2}\right)}{|1-\langle z, w\rangle|^{2}} .
$$

Bergman distance on $\mathbf{B}^{n}$ is given by the expression

$$
\rho(z, w)=\log \frac{|1-\langle z, w\rangle|+\sqrt{|z-w|^{2}+|\langle z, w\rangle|^{2}-|z|^{2}|w|^{2}}}{|1-\langle z, w\rangle|-\sqrt{|z-w|^{2}+|\langle z, w\rangle|^{2}-|z|^{2}|w|^{2}}} ;
$$

it can be also written in the following form, using $\varphi_{w}(z)$ :

$$
\rho(z, w)=\log \frac{1+\left|\varphi_{w}(z)\right|}{1-\left|\varphi_{w}(z)\right|} .
$$

Some general information and theorems on Bergman distance can be found in [4].

1.3. $\mathcal{M}$-invariant gradient. For a function $f \in C^{1}\left(\mathbf{B}^{n}\right)$, we define $\mathcal{M}$ invariant gradient by expression

$$
\widetilde{D} f(z)=D\left(f \circ \varphi_{z}\right)(0)
$$

where $D$ denotes the complex gradient

$$
D f(z)=\left\{\frac{\partial f}{\partial z_{j}}\right\}_{j=1}^{n}, \quad z_{j}=x_{j}+i y_{j}, \quad \frac{\partial f}{\partial z_{j}}=\frac{1}{2}\left(\frac{\partial f}{\partial x_{j}}+\frac{1}{i} \frac{\partial f}{\partial y_{j}}\right)
$$

and $\varphi_{z}$ is aforementioned automorphism of the unit ball $\mathbf{B}^{n}$. For $f \in \mathcal{H}\left(\mathbf{B}^{n}\right)$,

$$
|\widetilde{D} f(0)|=|D f(0)|=\left|f^{\prime}(0)\right| \text {. }
$$

The main property of $\mathcal{M}$-invariant gradient is the following invariance property

$$
\left|\widetilde{D}\left(f \circ \varphi_{z}\right)(0)\right|=|\widetilde{D} f(z)| \text {. }
$$

Basic properties about this notion can be found in [7] and [6]. We will use the following identity

$$
|\widetilde{D} f(z)|^{2}=\left(1-|z|^{2}\right)\left(|D f(z)|^{2}-|\langle D f(z), \bar{z}\rangle|^{2}\right), \quad f \in C^{1}\left(\mathbf{B}^{n}\right),
$$


see [6] for details. On the other hand, the real gradient $\nabla$ is defined by

$$
\nabla f(z)=\left\{\frac{\partial f}{\partial x_{j}}, \frac{\partial f}{\partial y_{j}}\right\}, \quad j=1,2 \ldots, n, \quad z_{j}=x_{j}+i y_{j} .
$$

For a function $f \in C^{1}\left(\mathbf{B}^{n}\right)$ we also define $\mathcal{M}$-invariant real gradient

$$
\widetilde{\nabla} f(z)=\nabla\left(f \circ \varphi_{z}\right)(0) .
$$

Analogous formulae hold for $\widetilde{\nabla}$ and $\widetilde{D}$, except for (1.5) where we have only

$$
|\widetilde{\nabla} f(0)|=|\nabla f(0)| \text {, }
$$

for $f \in C^{1}\left(\mathbf{B}^{n}\right)$.

1.4. Results. Schwarz's lemma is a fundamental result which states that for a holomorphic mapping $f: \mathbf{D} \rightarrow \mathbf{D}$, with $f(0)=0$, we have

$$
|f(z)| \leq|z| \text {. }
$$

This leads to another inequality which estimates the pseudohyperbolic distance between images of two points in terms of the pseudohyperbolic distance of points:

$$
\left|\frac{f(z)-f(w)}{1-\overline{f(z)} f(w)}\right| \leq\left|\frac{z-w}{1-\bar{z} w}\right|,
$$

and also the magnitude of the derivative at a point $z$ in terms of moduli of $z$ and its image $f(z)$ :

$$
\left|f^{\prime}(z)\right| \leq \frac{1-|f(z)|^{2}}{1-|z|^{2}}
$$

These basic results have been extended in numerous ways. The one that is of special interest for us is the following theorem.

Theorem 1. (Kalaj, [3]) If $f$ is a holomorphic mapping of the unit ball $\mathbf{B}^{n} \subset \mathbf{C}^{n}$ into $\mathbf{B}^{m} \subset \mathbf{C}^{m}$, then for $m \geq 2$

$$
\left|f^{\prime}(z)\right| \leq \frac{\sqrt{1-|f(z)|^{2}}}{1-|z|^{2}}, \quad z \in \mathbf{B}^{n}
$$

and for $m=1$ we have that

$$
\left|f^{\prime}(z)\right| \leq \frac{1-|f(z)|^{2}}{1-|z|^{2}}, \quad z \in \mathbf{B}^{n}
$$

Here $\left|f^{\prime}(z)\right|$ denotes the norm of the Frechet derivative of the mapping $f$. Let us recall that Frechet derivative of a holomorphic function $f: A \subset \mathbf{C}^{n} \rightarrow \mathbf{C}^{m}$ is the unique linear mapping $L=f^{\prime}(z): \mathbf{C}^{n} \rightarrow \mathbf{C}^{m}$ such that $f(z+h)-f(z)=$ $f^{\prime}(z) h+O\left(|h|^{2}\right)$.

Corollary 2. (Kalaj) Every holomorphic function $f: \mathbf{B}^{n} \rightarrow \mathbf{D}$ is a contraction with respect to the hyperbolic metric on both $\mathbf{B}^{n}$ and $\mathbf{D}$.

In section 2, we prove a refined version of Kalaj's theorem, using the so-called $\mathcal{M}$-invariant gradient $\widetilde{D}$.

Theorem 1.1. For each holomorphic function $f: \mathbf{B}^{n} \rightarrow \mathbf{D}$ we have

$$
|\widetilde{D} f(z)| \leq 1-|f(z)|^{2}, \quad z \in \mathbf{B}^{n},
$$


while for each holomorphic mapping $f: \mathbf{B}^{n} \rightarrow \mathbf{B}^{m}, m \geq 2$, we have

$$
|\widetilde{D} f(z)| \leq \sqrt{1-|f(z)|^{2}}, \quad z \in \mathbf{B}^{n} .
$$

Since $|\widetilde{D} f(z)| \geq\left(1-|z|^{2}\right)|D f(z)|$ (see section 2) this indeed improves Kalaj's theorem. In the case of the unit disc $\mathbf{D}$ we give a new technique, while for $n \geq 2$ we only refine the Kalaj's result.

Theorem 1.2. Every holomorphic function $f: \mathbf{B}^{n} \rightarrow \mathbf{D}$ is a contraction with respect to Bergman metric on $\mathbf{B}^{n}$ and $\mathbf{D}$.

As a direct consequence we will get

Corollary 1.3. (Schwarz-Pick inequality for several variables) For each holomorphic function $f: \mathbf{B}^{n} \rightarrow \mathbf{D}$ we have

$$
\left|\frac{f(z)-f(w)}{1-\overline{f(z)} f(w)}\right| \leq \frac{\sqrt{|z-w|^{2}+|\langle z, w\rangle|^{2}-|z|^{2}|w|^{2}}}{|1-\langle z, w\rangle|} .
$$

Note that for $n=1$, this is the classical Schwarz-Pick inequality.

The following result on plurisubharmonic functions from $\mathbf{B}^{n}$ to $(-1,1)$ can be found in[3]:

Theorem 3. Let $f$ be a pluriharmonic function from the unit ball $\mathbf{B}^{n} \subset \mathbf{C}^{n}$ to $(-1,1)$. Then the following sharp inequality holds

$$
|\nabla f(z)| \leq \frac{4}{\pi} \frac{1-|f(z)|^{2}}{1-|z|^{2}}, \quad z \in \mathbf{B}^{n}
$$

Using the $\mathcal{M}$-invariant gradient, we get the following refinement:

Theorem 1.4. For each pluriharmonic function $f: \mathbf{B}^{n} \rightarrow(-1,1)$ there holds the following inequality

$$
|\widetilde{\nabla} f(z)| \leq \frac{4}{\pi}\left(1-|f(z)|^{2}\right), \quad z \in \mathbf{B}^{n} .
$$

We also deal with Harnack's inequalities. It is well known that for positive harmonic functions $u: \mathbf{D} \rightarrow \mathbf{R}^{+}$we have

$$
\frac{1-|z|}{1+|z|} \leq \frac{u(z)}{u(0)} \leq \frac{1+|z|}{1-|z|} \quad \text { and } \quad|\nabla u(z)| \leq \frac{2 u(z)}{1-|z|}
$$

Dyakonov [2] proved the following lemma:

Lemma 4. Suppose $f \in \mathcal{H}\left(\mathbf{B}^{n}\right)$ is a function satisfying $0<|f(z)| \leq 1$ for all $z \in \mathbf{B}^{n}$. Then

$$
|D f(z)| \leq \frac{2|f(z)|}{1-|z|^{2}} \log \frac{1}{|f(z)|}
$$

Using the $\mathcal{M}$-invariant gradient form of Harnack's inequality, we prove the following refinement of Dyakonov's lemma:

Theorem 1.5. For each holomorphic function $f: \mathbf{B}^{n} \rightarrow \mathbf{D}$ without zeros in $\mathbf{B}^{n}$ we have

$$
|\widetilde{D} f(z)| \leq 2|f(z)| \log \frac{1}{|f(z)|}, \quad z \in \mathbf{B}^{n} .
$$

In section 3, we present a different proof, based only on Harnack's inequality, of the following Marković's theorem. 
Theorem 5. (Marković, [5]) Every harmonic function $f: \mathbf{H} \rightarrow \mathbf{R}^{+}$acts as a contraction with respect to hyperbolic metric considered on both $\mathbf{H}$ and $\mathbf{R}^{+}$.

We investigate the same problem in the upper half-space $\mathbf{H}_{n} \subset \mathbf{R}^{n}$ and the unit ball $\mathbf{B}_{n} \subset \mathbf{R}^{n}$ : Are positive harmonic functions contractions if we consider hyperbolic metric on $\mathbf{H}^{n}$ or $\mathbf{B}^{n}$ and $\mathbf{R}^{+}$? The answer is "no", see Section 3.

\section{Variations of the Schwarz lemma on the unit ball}

Using the Cauchy-Schwarz inequality we obtain:

$$
\begin{aligned}
|\widetilde{D} f(z)|^{2} & =\left(1-|z|^{2}\right)\left(|D f(z)|^{2}-|\langle D f(z), \bar{z}\rangle|^{2}\right) \\
& \geq\left(1-|z|^{2}\right)\left(|D f(z)|^{2}-|z|^{2}|D f(z)|^{2}\right) \\
& =\left(1-|z|^{2}\right)^{2}|D f(z)|^{2},
\end{aligned}
$$

and we have proved

$$
|\widetilde{D} f(z)| \geq\left(1-|z|^{2}\right)|D f(z)| \text {. }
$$

Proof of Theorem 1.1. (Case $m=1$.) Let us prove Theorem 1.1 in case $z=0$, i.e.

$$
|\widetilde{D} f(0)|=|D f(0)| \leq 1-|f(0)|^{2} .
$$

Let us fix $\zeta=\left(\zeta_{1}, \zeta_{2}, \ldots, \zeta_{n}\right) \in \partial \mathbf{B}^{n}$ and define a one-variable complex function

$$
g_{\zeta}(z)=f\left(\zeta_{1} z, \zeta_{2} z, \ldots, \zeta_{n} z\right), \quad z \in \mathbf{D} .
$$

Note that $g_{\zeta}$ maps $\mathbf{D}$ to $\mathbf{D}$ so we can apply Schwarz-Pick lemma to get

$$
\left|g_{\zeta}^{\prime}(0)\right| \leq 1-\left|g_{\zeta}(0)\right|^{2}
$$

Since

by choosing

$$
g_{\zeta}^{\prime}(z)=\zeta_{1} \frac{\partial f}{\partial z_{1}}(z)+\zeta_{2} \frac{\partial f}{\partial z_{2}}(z)+\cdots+\zeta_{n} \frac{\partial f}{\partial z_{n}}(z)
$$

we obtain the needed estimate

$$
\zeta_{i}=\frac{1}{|D f(0)|} \overline{\frac{\partial f}{\partial z_{i}}(0)},
$$

$$
|D f(0)| \leq 1-|g(0)|^{2}=1-|f(0)|^{2} .
$$

Applying (2.1) to $f \circ \varphi_{z}$ we get

$$
\left|\widetilde{D}\left(f \circ \varphi_{z}\right)(0)\right| \leq 1-\left|\left(f \circ \varphi_{z}\right)(0)\right|^{2} .
$$

The left-hand side, by (1.6), is equal to $|\widetilde{D} f(z)|$, so we have

$$
|\widetilde{D} f(z)| \leq 1-|f(z)|^{2} \text {. }
$$

(Case $m \geq 2$.) According to [3], we have

$$
|\widetilde{D} f(0)|=|D f(0)| \leq \sqrt{1-|f(0)|^{2}} .
$$

Applying this inequality to the function $f \circ \varphi_{z}$, we get:

$$
|\widetilde{D} f(z)|=\left|D\left(f \circ \varphi_{z}\right)(0)\right| \leq \sqrt{1-\left|f \circ \varphi_{z}(0)\right|^{2}}=\sqrt{1-|f(z)|^{2}} .
$$


Proof of Theorem 1.2. We have to prove $\rho(f(z), f(w)) \leq \rho(z, w)$ for a holomorphic $f: \mathbf{B}^{n} \rightarrow \mathbf{D}$. By Corollary 2, we have

$$
d(f(z), f(0)) \leq d(z, 0) .
$$

Since

$$
\rho(z, 0)=\log \frac{1+|z|}{1-|z|}=d(z, 0), \quad z \in \mathbf{B}^{n},
$$

and the Bergman and hyperbolic metrics coincide on $\mathbf{D}$, i.e.

$$
\rho(f(z), f(0))=d(f(z), f(0)),
$$

we conclude

$$
\rho(f(z), f(0)) \leq \rho(z, 0) .
$$

For prescribed $z, w \in \mathbf{B}^{n}$ there exist an automorphism $\varphi$ of the unit ball $\mathbf{B}^{n}$ such that $\varphi(0)=z, \varphi(a)=w$. Since $\varphi$ is an isometry in the Bergman metric, we have

$$
\rho(z, w)=\rho(\varphi(0), \varphi(a))=\rho(0, a) .
$$

Using (2.2) with $f \circ \varphi$ in place of $f$, we obtain

$$
\rho((f \circ \varphi)(0),(f \circ \varphi)(a)) \leq \rho(0, a)
$$

which gives

$$
\rho(f(z), f(w)) \leq \rho(0, a)=\rho(z, w) .
$$

From (1.1) and (1.2) it easily follows that $\left|T_{w}(z)\right| \geq\left|\varphi_{w}(z)\right|$, which leads to $\rho(z, w) \leq d(z, w)$. We see that Theorem 1.2 indeed refines Corollary 2 .

Proof of Corollary 1.3. By Theorem 1.2 and (1.3) we have

$$
\log \frac{1+\left|\frac{f(z)-f(w)}{1-\overline{f(z)} f(w)}\right|}{1-\left|\frac{f(z)-f(w)}{1-\overline{f(z)} f(w)}\right|} \leq \log \frac{|1-\langle z, w\rangle|+\sqrt{|z-w|^{2}+|\langle z, w\rangle|^{2}-|z|^{2}|w|^{2}}}{|1-\langle z, w\rangle|-\sqrt{|z-w|^{2}+|\langle z, w\rangle|^{2}-|z|^{2}|w|^{2}}}
$$

which implies

$$
\left|\frac{f(z)-f(w)}{1-\overline{f(z)} f(w)}\right| \leq \frac{\sqrt{|z-w|^{2}+|\langle z, w\rangle|^{2}-|z|^{2}|w|^{2}}}{|1-\langle z, w\rangle|} .
$$

Proof of the Theorem 1.4. The proof is similar to that of Theorem 1.1. For $z=0$ we have

$$
|\widetilde{\nabla} f(0)|=|\nabla f(0)| \leq \frac{4}{\pi}\left(1-|f(0)|^{2}\right) .
$$

Now, using this inequality for $f \circ \varphi_{z}$ we have, by $\widetilde{\nabla}$-version of (1.6):

$$
|\widetilde{\nabla} f(z)|=\left|\widetilde{\nabla}\left(f \circ \varphi_{z}\right)(0)\right| \leq \frac{4}{\pi}\left(1-\left|f \circ \varphi_{z}(0)\right|^{2}\right)=\frac{4}{\pi}\left(1-|f(z)|^{2}\right) .
$$

A consequence of the Harnack's inequality for a positive harmonic function $u$ in $\mathrm{D}$ is

$$
|\nabla u(z)| \leq \frac{2 u(z)}{1-|z|^{2}}
$$

Using Poisson representation we prove this for $z=0$ and extend the result to any $z \in \mathbf{D}$ using automorphisms $\varphi_{z}$. 
Proof of Theorem 1.5. Let us fix $\zeta \in \partial \mathbf{B}^{n}$ and apply (2.3) to positive harmonic function

$$
u(z)=\log \frac{1}{\left|F_{\zeta}(z)\right|},
$$

where $F_{\zeta}(z)=f\left(\zeta_{1} z, \zeta_{2} z, \ldots, \zeta_{n} z\right), z \in \mathbf{D}$ for point $z=0$ :

$$
\left|F_{\zeta}^{\prime}(0)\right| \leq 2\left|F_{\zeta}(0)\right| \log \frac{1}{\left|F_{\zeta}(0)\right|}=2|f(0)| \log \frac{1}{|f(0)|} .
$$

Next

by choosing

$$
F_{\zeta}^{\prime}(0)=\sum_{i=1}^{n} \zeta_{i} \frac{\partial f}{\partial z_{i}}(0)
$$

$$
\zeta_{i}=\frac{1}{|D f(0)|} \frac{\partial f}{\partial z_{i}}(0)
$$

where $|D f(0)|=\sqrt{\sum_{i=1}^{n}\left|\frac{\partial f}{\partial z_{i}}(0)\right|^{2}}$, we obtain

$$
|D f(0)| \leq 2|f(0)| \log \frac{1}{|f(0)|} .
$$

Because of (1.5) we get

$$
|\widetilde{D} f(0)| \leq 2|f(0)| \log \frac{1}{|f(0)|}
$$

Now, using inequality (2.4) for $f \circ \varphi_{z}, z \in \mathbf{B}^{n}$ in place of $f$, we obtain

$$
|\widetilde{D} f(z)|=\left|\widetilde{D}\left(f \circ \varphi_{z}\right)(0)\right| \leq 2\left|\left(f \circ \varphi_{z}\right)(0)\right| \log \frac{1}{\left|\left(f \circ \varphi_{z}\right)(0)\right|}=2|f(z)| \log \frac{1}{|f(z)|},
$$

and Theorem 1.5 is proved.

\section{Marković's theorem and counterexamples}

Here we give a new proof of Marković's theorem on positive harmonic functions on the upper half-plane and obtain counterexamples for higher-dimensional analogues.

We will use common Harnack inequality, which estimates the ratio of values of positive harmonic functions at an arbitrary point $z$ and at the zero:

$$
\frac{1-|z|}{1+|z|} \leq \frac{v(z)}{v(0)} \leq \frac{1+|z|}{1-|z|}
$$

3.1. Marković's theorem. For every harmonic function $v: \mathbf{D} \rightarrow \mathbf{R}^{+}$we can define harmonic function $u: \mathbf{H} \rightarrow \mathbf{R}^{+}$by

$$
u(z)=v(\varphi(z)), \quad \text { where } \quad \varphi(z)=\frac{z-i}{z+i},
$$

is a conformal mapping from $\mathbf{H}$ to $\mathbf{D}$. Conversely, for every harmonic $u: \mathbf{H} \rightarrow \mathbf{R}^{+}$ there is a harmonic $v: \mathbf{D} \rightarrow \mathbf{R}^{+}$given by $v(z)=u\left(\varphi^{-1}(z)\right)$, for which we have $u=v \circ \varphi$.

So, for $\zeta \in \mathbf{H}, \frac{\zeta-i}{\zeta+i} \in \mathbf{D}$, using (3.1) we obtain

$$
\frac{v\left(\frac{\zeta-i}{\zeta+i}\right)}{v(0)} \leq \frac{1+\left|\frac{\zeta-i}{\zeta+i}\right|}{1-\left|\frac{\zeta-i}{\zeta+i}\right|}=\frac{1+\left|\frac{\zeta-i}{\bar{\zeta}-i}\right|}{1-\left|\frac{\zeta-i}{\bar{\zeta}-i}\right|}, \quad \text { i.e. } \quad \frac{u(\zeta)}{u(i)} \leq \frac{1+\left|\frac{\zeta-i}{\bar{\zeta}-i}\right|}{1-\left|\frac{\zeta-i}{\bar{\zeta}-i}\right|}
$$


and after taking logarithms of both sides

$$
d(u(\zeta), u(i)) \leq d(\zeta, i)
$$

Let $\psi$ be a conformal automorphism of the upper half-plane which sends $\zeta$ and $i$ to $z$ and $w$, respectively. We have

$$
d(\zeta, i)=d(\psi(\zeta), \psi(i))=d(z, w)
$$

and

$$
d(u \circ \psi(\zeta), u \circ \psi(i))=d(u(z), u(w)),
$$

so (3.2) with $u \circ \psi$ instead of $u$ gives

$$
d(u(z), u(w)) \leq d(z, w) .
$$

So, Theorem 5 is proved.

Using the method of the above proof, one can show that any positive harmonic function from $\mathbf{D}$ to $(0,+\infty)$ is a contraction with respect to hyperbolic metrics on $\mathbf{D}$ and $\mathbf{R}^{+}$.

In the next subsection we provide counterexamples which show that these results do not extend to higher dimensions.

\subsection{Counterexamples for $n \geq 3$.}

Example 1. (The unit ball $\mathbf{B}_{n}$ ) The following Harnack's inequality for positive harmonic functions in the unit ball $\mathbf{B}_{n}$

$$
\frac{1-|x|}{(1+|x|)^{n-1}} \leq \frac{v(x)}{v(0)} \leq \frac{1+|x|}{(1-|x|)^{n-1}}
$$

gives us a clue for counterexamples in higher dimensions.

For $n \geq 3$, the hyperbolic metric in the unit ball $\mathbf{B}_{n}$ in $\mathbf{R}^{n}$ is given by

$$
d(x, y)=\cosh ^{-1}(1+\delta(x, y))=\log \left(1+\delta(x, y)+\sqrt{(1+\delta(x, y))^{2}-1}\right)
$$

where

$$
\delta(x, y)=\frac{2\|x-y\|^{2}}{\left(1-\|x\|^{2}\right)(1-\|y\|)^{2}}
$$

and $\|\cdot\|$ denotes the usual Euclidean norm.

So, for $y=0$ we have

$$
\begin{aligned}
& \delta(x, 0)=\frac{2\|x\|^{2}}{1-\|x\|^{2}} \\
& d(x, 0)=\log \left(1+\frac{2\|x\|^{2}}{1-\|x\|^{2}}+\sqrt{\left(\frac{2\|x\|^{2}}{1-\|x\|^{2}}\right)^{2}-1}\right)=\log \frac{1+\|x\|}{1-\|x\|} .
\end{aligned}
$$

If $u: \mathbf{B}_{n} \rightarrow \mathbf{R}^{+}$is harmonic, then

$$
d(u(x), u(0)) \leq d(x, 0) \quad \text { if and only if } \frac{u(x)}{u(0)} \leq \frac{1+\|x\|}{1-\|x\|} .
$$

But, for $\mathbf{1}=(1,0, \ldots, 0) \in \mathbf{R}^{n}$ and $n \geq 3$, the function

$$
u(x)=\frac{1-\|x\|^{2}}{\|\mathbf{1}-x\|^{n}}
$$


is positive harmonic, and setting $x=(t, 0, \ldots, 0), 0<t<1$, inequality (3.3) gives

$$
\frac{1-t^{2}}{(1-t)^{n}} \leq \frac{1+t}{1-t}
$$

that is $(1-t)^{2} \leq(1-t)^{n}$ which cannot hold for $n \geq 3$.

Example 2. (The upper half-space) In the case of the upper half-space $\mathbf{H}_{n} \subset \mathbf{R}^{n}$ for $n \geq 3$, the hyperbolic metric is given by

$$
d(x, y)=\cosh ^{-1}\left(1+\frac{\|x-y\|^{2}}{2 x_{n} y_{n}}\right),
$$

where $x=\left(x_{1}, x_{2}, \ldots, x_{n}\right), y=\left(y_{1}, y_{2}, \ldots, y_{n}\right)$ and $x_{n}, y_{n}>0$.

For $x=(0,0, \ldots, t), t>1$ and $y=(0,0, \ldots, 1)$, we have

$$
\delta(x, y)=1+\frac{\|x-y\|^{2}}{2 x_{n} y_{n}}=1+\frac{(t-1)^{2}}{2 t}=\frac{t^{2}+1}{2 t}
$$

and

$$
d(x, y)=\log \left(\delta(x, y)+\sqrt{\delta(x, y)^{2}-1}\right)=\log \left(\frac{t^{2}+1}{2 t}+\sqrt{\left(\frac{t^{2}+1}{2 t}\right)^{2}-1}\right)=\log t
$$

so $d(u(x), u(y)) \leq d(x, y)$ is equivalent to

$$
\frac{u(y)}{u(x)} \leq t
$$

Choosing positive harmonic function $u(x)$ to be

we obtain

$$
u(x)=\frac{x_{n}}{\|x\|^{n}}
$$

$$
\frac{u(y)}{u(x)}=\frac{1}{t^{1-n}}=t^{n-1}
$$

which cannot be smaller than $t$ for $n \geq 3$, for $t>1$.

Acknowledgements. The author thanks to Miroslav Pavlović, Miloš Arsenović and Nikola Milinković for helpful comments and corrections of some mistakes.

\section{References}

[1] Ahlfors, L.: Möbius transformations in several dimensions. - Lecture Notes at University of Minnesota, 1981.

[2] Dyakonov, K. M.: Functions in Bloch-type spaces and their moduli. - Ann. Acad. Sci. Fenn. Math. 41:2, 2016, 705-712.

[3] KalaJ, D.: Schwarz lemma for holomorphic mappings in the unit ball. - arXiv:1504.04823, 2015.

[4] Krantz, S. G.: Function theory of several complex variables. - Amer. Math. Soc., 2001.

[5] Marković, M.: On harmonic functions and the hyperbolic metric. - Indag. Math. 26:1, 2015, 19-23.

[6] Pavlović, M.: Inequalities for the gradient of eigenfunctions of the invariant Laplacian in the unit ball. - Indag. Math. 2:1, 1991, 89-98.

[7] Rudin, W.: Function theory in the unit ball of $\mathbf{C}^{n}$. - Springer, 1980.

Received 19 December 2016 • Accepted 8 September 2017 